\title{
BTZ entropy from topological M-theory
}

\author{
Javier Chagoya $\odot,{ }^{1, *}$ Graciela Reyes-Ahumada $\odot,{ }^{2}$ and M. Sabido $\oplus^{3, \dagger}$ \\ ${ }^{1}$ Unidad Académica de Física, Universidad Autónoma de Zacatecas, 98060 Zacatecas, México \\ ${ }^{2}$ Conacyt-Universidad Autónoma de Zacatecas, 98060 Zacatecas, México \\ ${ }^{3}$ Departamento de Física de la Universidad de Guanajuato, \\ A.P. E-143, C.P. 37150 León, Guanajuato, México
}

(Received 20 November 2020; accepted 12 March 2021; published 9 April 2021)

\begin{abstract}
By determining the relation between topological M-theory and the Chern-Simons actions for a gauge field constructed from the Lie algebra of either $S L(2, \mathbb{R}) \times S L(2, \mathbb{R})$ or $S L(2, \mathbb{C}) \times S L(2, \mathbb{C})$, depending on the sign of the space-time curvature, we show that the standard and exotic actions of three-dimensional gravity can be recovered from topological M-theory. With this result, we provide a concrete realization of a conjecture by Dijkgraaf et al. stating that the partition function of topological M-theory is equivalent to the partition function of a black hole in a related theory. We do this for the standard and exotic BTZ black holes in three-dimensional gravity.
\end{abstract}

DOI: 10.1103/PhysRevD.103.084009

\section{INTRODUCTION}

One of the most useful tools for understanding gravitational interaction is three-dimensional gravity. Not only has $2+1$ gravity been quantized, it has other remarkable features that are of great value as a guide to understanding the foundations of gravity. Some of these features can be easily derived from the fact that it can be written as a ChernSimons action [1]. And although $2+1$ gravity is topological and therefore might seem physically unrealistic (it lacks propagating degrees of freedom), there is a black hole solution known as the Bañados-Teitelboim-Zanelli (BTZ) black hole [2]. The BTZ solution is asymptotically anti-de Sitter and has no singularity, but it has many of the features of the Kerr black hole. It has an event horizon and an inner horizon for the rotating case, also the thermodynamic properties are analogous to four-dimensional black holes. Interestingly the BTZ solution represents a black hole solution with mass $m$ and angular momentum $j$. It is a solution to any $2+1$ gravity model that admits antide Sitter vacuum, with any linear combination of the parameters $m$ and $j$. When the role of mass and angular momentum is reversed, the resulting black hole is known as an exotic BTZ black hole. The entropy of the BTZ black hole is in agreement with Hawking-Bekenstein entropy, but for the exotic case the entropy is related to the inner

\footnotetext{
javier.chagoya@fisica.uaz.edu.mx msabido@fisica.ugto.mx
}

Published by the American Physical Society under the terms of the Creative Commons Attribution 4.0 International license. Further distribution of this work must maintain attribution to the author(s) and the published article's title, journal citation, and DOI. Funded by SCOAP ${ }^{3}$. horizon. This appalling contradiction was resolved in [3] by considering that the BTZ is a solution to the standard action and the exotic BTZ is a solution to the exotic action, and consequently the entropy must be given by

$$
S=\frac{\pi}{2 G}\left(\alpha r_{+}+\gamma r_{-}\right) .
$$

The standard and exotic actions are the two independent actions that are derived in the Chern-Simons formulation of $2+1$ gravity [1,4].

The description of the gravitational field in terms of gauge fields or $p$-forms has been continuously developed. In these theories the metric does not appear explicitly but it is reconstructed from the dynamical fields under consideration. These descriptions are referred to as form theories of gravity. Some of these form theories, including Chern-Simons (CS) three-dimensional (3d) gravity and the $\mathrm{A}$ and $\mathrm{B}$ models of topological strings can be unified in a seven-dimensional space-time, $X$, through the topological M-theory (TMT) proposed by Dijkgraaf et al. [5]. Essential in this theory is the volume form $V$ constructed from an invariant $p$-form whose existence is characteristic of special holonomy manifolds. The study of manifolds admitting stable nondegenerate forms is an interesting topic by itself, see for example [6] for a classification of all stable forms on $\mathbb{R}^{n}$. In particular, for seven dimensions, there are two nontrivial $p$-forms invariant under the holonomy group $G_{2}$, one of which is a 3 -form and the other a 4 -form. The same is true for the stable $p$-forms invariant under the dual group $\tilde{G}_{2}$. Using the 3 -form $\Phi$ with holonomy in $G_{2}$, Dijkgraaf et al. showed that the equations of motion for $2+1$ gravity are recovered under a convenient partition of $X$. However, it is known that for 
nonvanishing cosmological constant, $\lambda$, there are two classically equivalent actions to describe gravity in $2+1$ dimensions and they are known as standard and exotic actions $[1,4]$. As these two actions are classically equivalent, they cannot be distinguished from the equations of motion derived in [5] (this is not an issue for the 4d case, because unlike $2+1$ gravity, there is only one action). In [7], the authors obtain these actions from TMT. This result opens up the possibility to apply the formalism and ideas of TMT to several models of $2+1$ gravity that are built in terms of the CS actions. We consider that CS gravity can be used to better understand TMT. In particular we will explore the relation between the partition function of the Hitchin volume functional and the partition function of black holes [5].

In [8], it is conjectured that the partition function of a $4 d$ Bogomol'nyi-Prasad-Sommerfield (BPS) black hole in a Calabi-Yau compactification of type II superstring theory is related to the topological string partition function by

$$
Z_{\mathrm{BH}}=\left|Z_{\mathrm{top}}\right|^{2} .
$$

Furthermore, it is pointed out that the topological partition function can be interpreted as a wave function. They conjectured that the topological string wave function computes the partition function of BPS black hole states in $4 \mathrm{~d}$ and is given by

$$
Z_{\mathrm{BH}}=\left|\psi_{\text {top }}\right|^{2} .
$$

Additionally in [5] it is proposed that the partition function $Z_{H}$ associated with the holomorphic volume functional $V_{H}$ is related to the partition function of the B model of topological strings $Z_{B}$ and its complex conjugate $\bar{Z}_{B}$. More precisely, they argue that $Z_{H}$ is the Wigner function of the topological string $B$ model. These conjectures are related to black holes and topological string theory. Considering that topological string theory is contained in TMT, we can ask ourselves if these conjectures can be realized in the context of TMT.

In this work we present a realization of these ideas, but in the context of $3 \mathrm{~d}$ gravity. As shown in [5] at the level of the equations of motion and in [7] at the level of the action, $3 \mathrm{~d}$ gravity is contained in TMT as a particular splitting of the $7 \mathrm{~d}$ manifold. In order to give a concrete example of the relation between $Z_{H}$ and the black hole entropy we consider an extremal BTZ black hole, compute its volume form in terms of the $2+1$ dimensional standard and exotic actions for gravity, then we obtain $Z_{H}$, and finally we compare it to the norm of the wave function for the same black hole [9]. Then we can write the conjecture as

$$
Z_{H}=Z_{\mathrm{BH}}=\left|\psi_{\mathrm{top}}\right|^{2}
$$

where $Z_{H}$ is the partition function of the Hitchin functional. As a side result, using TMT we can study the result in [3] for the entropy of a BTZ black hole. We find that the entropy of the BTZ black hole calculated from the TMT is related to the volume functional. When considering the linear combination of the two volume functionals, we reproduce the result in [3] for the entropy.

The organization of this work is as follows. First, we review and formalize the derivation of the standard and exotic actions for $2+1$ gravity from TMT and construct the topological partition function. Then, we also review the BTZ black hole solutions and its partition function obtained from canonical quantization. Finally, we show how these results are related.

\section{STABLE FORMS IN 7D}

In this section we study the relation between invariant stable forms and structures on a 7d Riemannian manifold, $\mathbb{R}^{7}$. To understand the geometric structures defined by stable forms, we need to study the isotropy subgroup of such forms under the action of the general linear group $G L(7)$. We start by recalling the structure on $\mathbb{R}^{7}$. Later we use such construction to understand the case of a manifold $X$.

Let $V$ be a real $7 \mathrm{~d}$ vector space with basis $\left\{e_{i}\right\}$ and consider the space of 3-forms $\wedge^{3} V^{*}$. A form $\omega$ in $\wedge^{3} V^{*}$ can be written as

$$
\omega=\sum_{i, j, k=1}^{7} a_{i j k} e^{i j k},
$$

where $e^{i j k}=e^{i} \wedge e^{j} \wedge e^{k}$ and $\left\{e^{i}\right\}$ is a basis for $V^{*}$. Consider the group $G=G L(7)$ of automorphisms of $V$. There is a natural action $G \curvearrowright \wedge^{3} V^{*}$ and it is known that there are two distinguished orbits given by this action, namely

$$
\begin{gathered}
G \cdot \omega_{1}, \\
G \cdot \omega_{2},
\end{gathered}
$$

where $\omega_{i}$ is the form defined as

$$
\begin{aligned}
& \omega_{1}=e^{123}-e^{145}+e^{167}+e^{246}+e^{257}+e^{347}-e^{356}, \\
& \omega_{2}=e^{123}+e^{145}-e^{167}+e^{246}+e^{257}+e^{347}-e^{356} .
\end{aligned}
$$

Each form corresponds to an isotropy group, the Lie group

$$
G_{\omega_{1}}=G_{2}, \quad G_{\omega_{2}}=\widetilde{G}_{2} .
$$

It is proved in [10] that $G_{2}$ is compact, connected, simple, simply connected, 14-dimensional and it fixes the Euclidean metric $g_{1}=\sum\left(x^{i}\right)^{2}$ where $x=x^{i} e_{i}$ and $y=y^{i} e_{i}$ induced by 
$\langle x, y\rangle_{\omega_{1}}=x^{1} y^{1}+x^{2} y^{2}+x^{3} y^{3}+x^{4} y^{4}+x^{5} y^{5}+x^{6} y^{6}+x^{7} y^{7}$.

$G_{2}$ also preserves the orientation of the forms $\omega_{1}$ and $* \omega_{1}$ with respect to $g_{1}$, and $G_{2}$ is isomorphic to the group of automorphisms of the octonions.

There are analogous results for the group $\widetilde{G}_{2}$; this group preserves $\omega_{2}, * \omega_{2}$, the metric induced by

$\langle x, y\rangle_{\omega_{2}}=x^{1} y^{1}+x^{2} y^{2}+x^{3} y^{3}-x^{4} y^{4}-x^{5} y^{5}-x^{6} y^{6}-x^{7} y^{7}$,

and it is the noncompact dual of $G_{2}$. It is also connected, of dimension 14, and simple.

In this case the natural identification

$$
G \cdot \omega_{i}=G / G_{\omega_{i}}
$$

is in fact a diffeomorphism. Since $\operatorname{dim}(G)=49$ and $\operatorname{dim}\left(G_{2}\right)=\operatorname{dim}\left(\widetilde{G}_{2}\right)=14$ then the dimension of these orbits $\operatorname{dim}\left(G \cdot \omega_{i}\right)=49-14=35$ coincides with the dimension of the ambient space $\operatorname{dim}\left(\wedge^{3} V^{*}\right)=35$ and we conclude as in [10] that both orbits are open and the forms $\omega_{1}$ and $\omega_{2}$ are stable. In [6], the authors show that the forms $\omega_{1}, \omega_{2}$ are essentially the unique stable forms, in the sense that any stable form $\omega \in \wedge^{3} V^{*}$ is either in the orbit of $\omega_{1}$ or $\omega_{2}$.

The scenario we study in this paper is the case when $X$ is a complete $7 \mathrm{~d}$ Riemannian manifold, $x \in X$ is a point and $V=T_{x} X$. A stable form induces a $G_{\omega_{i}}$-structure on $X$, as follows (see [11]):

Consider the fiber bundle $\wedge^{3} T^{*} X$ and the open subbundle $\mathcal{P}^{i}(X)$ with fiber

$$
\mathcal{P}_{x}^{i}=\left\{\omega \in \wedge^{3} V^{*} \mid \exists f: V \rightarrow \mathbb{R}^{7} \quad \text { with } \quad f^{*}\left(\omega_{i}\right)=\omega\right\},
$$

where in the last definition $f$ is an oriented isomorphism. From the previous discussion $\mathcal{P}_{x}^{3} \cong G \cdot \omega_{i}$. Fix a form $\omega$ over $X$ such that $\left.\omega\right|_{p} \in \mathcal{P}_{x}^{i}=g \cdot \omega_{i}$ and consider the frame bundle $F$ of $X$ with fiber

$$
F_{x}=\left\{f \mid f: V \rightarrow \mathbb{R}^{7} \text { is an isometry }\right\} .
$$

Let $Q$ be the principal sub-bundle of $F$ whose fiber consists in isomorphisms preserving $\omega$. Hence the fiber is $Q_{x} \cong G_{\omega_{i}}$ and $\omega$ determines $Q$ which defines a $G_{\omega_{i}}$-structure on $X$, preserving the metric $g_{\omega}$ induced by the inner product

$$
\langle x, y\rangle_{\omega}=g \cdot\langle x, y\rangle_{\omega_{i}} .
$$

There is a converse for this construction: given an oriented $G_{\omega_{i}}$-structure we can define a metric $g$, a 3 -form $\omega$, and $* \omega$ requiring that the corresponding metric is preserved by the action of $G_{\omega_{i}}$.

Let $X$ be a Riemannian $7 \mathrm{~d}$ manifold with a $G_{2}$ structure $(\omega, g)$ and denote as $\nabla_{g}$ the Levi-Civita connection associated with $g$. Let $\nabla_{g} \omega$ be the torsion of this $G_{2}$ structure. We say that $(\omega, g)$ is torsion-free if $\nabla_{g} \omega=0$. Finally define a $G_{2}$ manifold as a triplet $(X, \omega, g)$ such that $(\omega, g)$ is torsion-free.

Consider a $G_{2}$ manifold $X$. The existence of a $G_{2}$ holonomy metric is equivalent to the existence of a 3 -form $\Phi$ satisfying as in [5]

$$
\begin{aligned}
d \Phi & =0, \\
d_{* \Phi} \Phi & =0 .
\end{aligned}
$$

A stable 3 -form can be written in terms of a $7 \mathrm{~d}$ vielbein as

$$
\Phi=\sum_{i, j, k=1}^{7} \Psi_{i j k} e^{i} e^{j} e^{k},
$$

where $\Psi_{i j k}$ are the structure constants of the imaginary octonions. There are analogous constructions for stable forms on a $\widetilde{G}_{2}$ manifold, since the orbits of $\omega_{1}, \omega_{2}$ correspond with the holonomy groups $G_{2}$ and $\widetilde{G}_{2}$ respectively.

In order to define a volume on a $G_{\omega_{i}}$ manifold $X$ consider a 3-form $\Phi$ on $X$ as before, invariant by the corresponding holonomy group and define a volume as

$$
V_{7}(\Phi)=\int_{X} \Phi \wedge_{* \Phi} \Phi .
$$

As above since in the $7 \mathrm{~d}$ case there are only two open orbits of maximal dimension, it is natural to consider only forms in these orbits to get a notion of genericity as in [5].

\section{3D GRAVITY FROM TOPOLOGICAL M-THEORY}

In [5], Dijkgraaf et al. introduced a notion for TMT in 7d with the property that it seems to unify several lower dimensional topological models. In particular, they find a dimensional reduction that recovers the equations of motion of $2+1$ gravity from the volume of the $7 \mathrm{~d}$ manifold $X$ discussed in the previous section. A similar construction was given by Bryant et al. [10], where starting from a rank4 spin bundle $\mathbf{S}$ over a $3 \mathrm{~d}$ space of constant curvature (space form), a 3-form $\Phi$ satisfying $d \Phi=d_{* \Phi} \Phi=0$ is constructed by making use of the structure equations for a manifold with constant sectional curvature $\kappa \equiv 4 \Lambda$, i.e.,

$$
\begin{gathered}
d e=-A \wedge e-e \wedge A, \\
d A=-A \wedge A-\Lambda e \wedge e
\end{gathered}
$$

where $\left\{e^{1}, e^{2}, e^{3}\right\}$ is a basis of the tangent space at a point of the 3-manifold, and $A$ is a Levi-Civita connection 1 -form. As [5,10] point out, a 3-form that generalizes $\omega_{1}$ (8) can satisfy the conditions $d \Phi=d_{* \Phi} \Phi=0$ in some 
special cases. In order to write down this 3 -form $\psi$ it is convenient to introduce first a set of local coordinates on the $4 \mathrm{~d}$ fiber. Let $y_{i}$ be those coordinates; we define $r=y_{i} y^{i}$. Notice that this is $S O(4)$ invariant. With the following 2-forms,

$$
\begin{aligned}
& \Sigma^{5}=e^{12}-e^{34}, \\
& \Sigma^{6}=e^{13}-e^{42}, \\
& \Sigma^{7}=e^{14}-e^{23},
\end{aligned}
$$

we can write the 3-form $\Phi$ that satisfies $d \Phi=d_{* \Phi} \Phi=0$ as

$$
\Phi=f^{3}(r) e^{567}+f(r) g^{2}(r) e^{m} \wedge \Sigma^{m} .
$$

Since $f$ and $g$ depend only on $r, \Phi$ preserves the $S O(4)$ invariance of $\omega_{1}$. Remembering that $S O(4)$ is a subgroup of $G_{2}$, and by the discussion of the previous section, the fact that $\Phi$ is $S O(4)$ invariant is a good indicator that it can define a $G_{2}$ structure-thus satisfying the required equations. The local coordinates $y_{i}$ are also used to define a basis of 1 -forms in the fiber direction as

$$
\alpha=d y-y A \text {. }
$$

The four components of $\alpha$ are identified as a local basis on the fiber, $\alpha^{i}=e^{i}, i=4,5,6,7$. As a consequence of Eqs. (14), these 1-forms satisfy

$$
d \alpha=-\alpha \wedge A+(\kappa / 4) y \omega \wedge \omega .
$$

Using Eqs. (14), (18), and

$$
{ }_{* \Phi} \Phi=-\frac{1}{6} g^{4} \Sigma_{m} \wedge \Sigma^{m}+\frac{1}{2} f^{2} g^{2} \epsilon^{m n p} e^{m} \wedge e^{n} \wedge \Sigma^{p},
$$

in [12] it is shown that the equations $d \Phi=d_{* \Phi} \Phi=0$ hold if

$$
\begin{aligned}
& f(r)=\sqrt{3 \Lambda}(1+r)^{1 / 3}, \\
& g(r)=2(1+r)^{-1 / 6} .
\end{aligned}
$$

Conversely, the authors of [5] start with $d \Phi=d_{* \Phi} \Phi=0$ and verify that the above assumptions for $f(r)$ and $g(r)$ lead to the structure equations, (14), i.e., in their interpretation, the equations of motion for $3 \mathrm{~d}$ gravity arise from the equations for a 3 -form with $G_{2}$ holonomy. If these equations of motion are recovered from such a 3-form $\Phi$, it is natural to look for a Lagrangian for $\Phi$ that encompasses the main points of the derivations above and reduces to the known Lagrangians for $3 \mathrm{~d}$ gravity. This Lagrangian is given precisely in terms of the volume form discussed around Eq. (13). In order to convert Eq. (13) into an expression that we can recognize as the action for $2+1$ gravity, we perform the following steps. First, we rewrite the integrand $\Phi \wedge_{* \Phi} \Phi$ using the antisymmetry of the wedge product and of the Levi-Civita tensor, obtaining

$$
V_{7}(\Phi)=\int_{X} \frac{40}{3}(3 \Lambda)^{3 / 2}(1+r)^{1 / 3} e^{567} \wedge \Sigma_{i} \wedge \Sigma^{i} .
$$

Now, let $\Sigma$ be the curvature of a connection $\alpha$, i.e.,

$$
\Sigma_{5}=d \alpha_{5}+2 \alpha_{6} \alpha_{7}
$$

and cyclically for the others. Later on we will relate this $\alpha$ to the connection 1-form $A$. Notice that this is compatible with the equations (15) that express $\Sigma^{i}$ in a local orthonormal basis [13]. Using again the properties of the wedge product, and noticing that as a consequence of the structure equations (14) we have $d\left(e^{567}\right)=0$ [12], the volume $V_{7}$ can be written as

$$
\begin{aligned}
V_{7}(\Phi)= & \int_{X} \frac{40}{3}(3 \Lambda)^{3 / 2}(1+r)^{1 / 3} d\left[e ^ { 5 6 7 } \wedge \left(\alpha_{i} \wedge d \alpha_{i}\right.\right. \\
& \left.\left.+\frac{2}{3} \epsilon^{i j k} \alpha_{i} \alpha_{j} \alpha_{k}\right)\right] .
\end{aligned}
$$

The argument of the differential does not depend on $r$, therefore, by an appropriate choice of coordinates, its prefactor can be integrated out so that it becomes a global factor of a $6 \mathrm{~d}$ integral. We can further reduce these dimensions by using Stokes's theorem, obtaining ${ }^{1}$

$$
V_{7}(\Phi) \propto \int_{X^{5}} e^{567} \wedge\left(\alpha_{i} \wedge d \alpha_{i}+\frac{2}{3} \epsilon^{i j k} \alpha_{i} \alpha_{j} \alpha_{k}\right)
$$

Finally, since the argument of the integral only depends on quantities defined over the 3-manifold $\mathcal{M}$ with basis $\left\{e^{5}, e^{6}, e^{7}\right\}$, the volume can be expressed as

$$
V_{7}(\Phi) \sim \int_{\mathcal{M}} e^{567} \wedge\left(\alpha_{i} \wedge d \alpha_{i}+\frac{2}{3} \epsilon^{i j k} \alpha_{i} \alpha_{j} \alpha_{k}\right) .
$$

Expanding the wedge product in components, relabeling the internal indices as $(a, b, c)$ and using $(i, j, k)$ for the spacetime indices, we get

$$
V_{7}(\Phi) \sim \int_{\mathcal{M}} \epsilon^{i j k}\left(2 \alpha_{i}^{a} \wedge \partial_{j} \alpha_{k}^{a}+\frac{2}{3} \epsilon_{a b c} \alpha_{i}^{a} \alpha_{j}^{b} \alpha_{k}^{c}\right) .
$$

This is the Chern-Simons action. At this point it is convenient to notice that the 2-forms $\Sigma$ are anti-self-dual,

\footnotetext{
${ }^{1}$ We have to be careful with the notation: all $p$-forms are integrated over $p$-dimensional manifolds. If the dimensions of the integral and the order of the $p$-form obtained by counting wedge products does not match, this means that one of the differentials $d x^{i}$ has been integrated out, and we have to remember this when writing the form in component notation.
} 
i.e., ${ }^{*} \Sigma^{i}=-\Sigma^{i}$. For this reason, we rename it as ${ }^{-} \Sigma^{i}$, with associated connection ${ }^{-} \alpha_{i}$, and we also rename the form $\Phi$ given in Eq. (16) as $-\Phi$. Now we are ready to see the relevance of the discussion of the previous section. The form ${ }^{-} \Phi$ is constructed out of the stable form $\omega_{2}$ presented in Eq. (9). However, we have seen that the volume form can also be constructed in terms of $\omega_{1}$, Eq. (8). Moreover, these two possibilities, $\omega_{1}$ and $\omega_{2}$, are unique in the sense discussed in the previous section. With these considerations in mind, we construct a volume form for each of the 3-forms

$$
\begin{aligned}
& { }^{-} \Phi=f^{3}(r) e^{567}+f(r) g^{2}(r) e^{m} \wedge{ }^{-} \Sigma^{m}, \\
& { }^{+} \Phi=f^{3}(r) e^{567}+f(r) g^{2}(r) e^{m} \wedge^{+} \Sigma^{m},
\end{aligned}
$$

where ${ }^{+} \Sigma^{m}$ are the self-dual 2-forms

$$
\begin{aligned}
& { }^{+} \Sigma^{5}=e^{12}+e^{34}, \\
& { }^{+} \Sigma^{6}=e^{13}+e^{42}, \\
& { }^{+} \Sigma^{7}=e^{14}+e^{23},
\end{aligned}
$$

and $r$ is defined in the same way as described before. When $f(r)=g(r)=1,-{ }^{-} \Phi,{ }^{+} \Phi$ are equivalent to $\omega_{2}$ and $\omega_{1}$, respectively. The 4 -forms associated with ${ }^{-} \Phi$ and ${ }^{+} \Phi$ are

$$
\begin{aligned}
{ }_{* \Phi}^{\mp} \Phi= & \mp \frac{1}{6} g^{4 \mp} \Sigma_{m} \wedge{ }^{\mp} \Sigma^{m} \\
& \pm \frac{1}{2} f^{2} g^{2} \epsilon^{m n p} e^{m} \wedge e^{n} \wedge{ }^{\mp} \Sigma^{p} .
\end{aligned}
$$

We can use either of ${ }^{ \pm} \Phi$ to construct the volume of the 7-manifold $X$,

$$
V^{ \pm} \equiv V_{7}\left({ }^{ \pm} \Phi\right)=\int_{X}{ }^{ \pm} \Phi \wedge_{* \Phi}{ }^{ \pm} \Phi .
$$

By the same steps of the previous section, $V_{7}$ can be written as

$V^{ \pm} \sim \int_{\mathcal{M}} \epsilon^{i j k}\left(2^{ \pm} \alpha_{i}^{a} \wedge \partial_{j}{ }^{ \pm} \alpha_{k}^{a}+\frac{2}{3} \epsilon_{a b c}{ }^{ \pm} \alpha_{i}^{a \pm} \alpha_{j}^{b \pm} \alpha_{k}^{c}\right)$,

where ${ }^{+} \alpha^{i}$ is the connection associated with ${ }^{+} \Sigma^{i}$. Thus, we have found two Chern-Simons actions derivable from the volume of a 7-manifold that admits two special stable forms. Now we want to understand how these two actions are related to $2+1$ gravity. From the results of $[5,12]$, we know that the equations of motion arising from the volume of ${ }^{-} \Phi$ are those of $2+1$ gravity with a cosmological constant. Since $V\left({ }^{+} \Phi\right)$ describes the same volume as $V\left({ }^{-} \Phi\right)$, the $3 \mathrm{~d}$ equations of motion derived from both actions have to coincide. This is remarkably similar, and consistent, with the results of [1], where it is shown that there are two 3d actions, named standard and exotic, that lead to the same equations of motion that we are interested in. Moreover, it is proven that these actions can be written in terms of the Chern-Simons actions Eq. (32) by setting

$$
{ }^{ \pm} \alpha_{i}^{a}=A_{i}^{a} \pm \sqrt{\lambda} e_{i}^{a},
$$

where $A_{i}$ and $e_{i}$ are the fields introduced around Eq. (14). The combinations

$$
\begin{gathered}
I_{\mathrm{st}}=\frac{{ }^{+} I-{ }^{-} I}{4 \sqrt{\lambda}}, \\
I_{\mathrm{ex}}=\frac{{ }^{+} I+{ }^{-} I}{2},
\end{gathered}
$$

where ${ }^{ \pm} I$ are the integrals in Eq. (32), give respectively the standard and exotic actions.

Now we can reinterpret the standard and exotic actions in terms of the volume functional as

$$
\begin{aligned}
I_{\mathrm{st}} & =\frac{h^{+} V^{+}-h^{-} V^{-}}{4 \sqrt{\lambda}}, \\
I_{\mathrm{ex}} & =\frac{h^{+} V^{+}+h^{-} V^{-}}{2},
\end{aligned}
$$

where $h^{ \pm}$are the inverses of the proportionality factors in Eq. (32). In this way, we can see the standard and exotic actions as two different combinations of pieces of the volume of the 7-manifold X. Applications of the ideas developed so far to the Immirzi ambiguity in $3 \mathrm{~d}$ gravity have been presented in [7]. In the next section we explore the entropy of the BTZ black hole from the point of view of TMT and we discuss the relation of our results to the conjecture $Z_{\mathrm{BH}}=\left|Z_{\mathrm{top}}\right|^{2}$.

\section{BTZ BLACK HOLE: PARTITION FUNCTION}

Using the results described above we can provide evidence that the conjecture discussed around Eq. (2) also applies for $G_{2}$ manifolds and $3 \mathrm{~d}$ black holes, i.e., that in general, the partition function of a theory with action defined by a Hitchin functional is related to the partition function for a BPS black hole in the gravitational theory allowed by the $p$-forms used to construct the Hitchin functional. The possibility that the relation between BPS objects and form theories of gravity extends to G2 manifolds was hinted in [5]. However, it was only studied for 4d and $5 \mathrm{~d}$ black holes embedded in a $6 \mathrm{~d} S U(3)$ manifold. The $4 \mathrm{~d}$ BPS black hole is constructed in a Calabi-Yau compactification of type II string theory $[5,8]$ and the partition function is related to the $6 \mathrm{~d}$ topological sting model derived in TMT. The $5 \mathrm{~d}$ black hole is derived from M-theory compactified in the Calabi-Yau [5]. The 4d and 5d black holes are not solutions to the $4 \mathrm{~d}$ or $5 \mathrm{~d}$ form theories of 
gravity derived from TMT. In contrast, for the $3 \mathrm{~d}$ case the BTZ black hole is a solution to the $3 \mathrm{~d}$ form theory of gravity derived from TMT. In this work we show explicitly that the partition function of the BTZ black hole is recovered from the partition function associated with the volume $V_{7}$. Given the different ways of writing down $V_{7}$ either in terms of $V^{+}, V^{-}$or both, one could think that the result only applies to the extremal case, which turns out to be associated with the situation where we demand that the linear combinations of $V^{+}$and $V^{-}$, for instance $I_{\mathrm{st}}$ and $I_{\mathrm{ex}}$, preserve a given multiple of $V_{7}$; but as we argue below, the partition function obtained from TMT correctly gives the $\mathrm{BH}$ partition function even away from the extremal case.

In the case of TMT, the total space $X$ is $7 \mathrm{~d}$ and as we shown in the previous sections, its volume can be constructed with either of the 3 -forms ${ }^{+} \Phi$ and $-\Phi$. A certain combination of these volumes, Eq. (34), results in the standard action for $3 \mathrm{~d}$ gravity. In this theory, a black hole solution is given by the BTZ space-time [2], whose metric can be written as

$$
d s^{2}=-N^{2} d t^{2}+N^{-2} d r^{2}+r^{2}\left(N^{\phi} d t+d \phi\right)^{2},
$$

where the lapse $N$ and shift $N^{\phi}$ are

$$
\begin{gathered}
N=\left(-M+\frac{r^{2}}{\ell^{2}}+\frac{J^{2}}{4 r^{2}}\right)^{1 / 2}, \\
N^{\phi}=-\frac{J}{2 r^{2}} .
\end{gathered}
$$

The integration constants $M$ and $J$ are interpreted respectively as the mass and angular momentum of the black hole, and $\ell$ is related to the cosmological constant of the theory by $\ell^{-2}=\Lambda / 3$. The lapse function vanishes at two distinct values of $r$, defining two coordinate singularities, $r_{ \pm}$,

$$
r_{ \pm}=\frac{1}{2}(\sqrt{\ell(\ell M+J)} \pm \sqrt{\ell(\ell M-J)}) .
$$

When $J=0$ only $r_{+}$is different from zero, and in the extremal case $J=M \ell$ the two horizons coincide. The entropy of the BTZ black hole can be computed by different methods, for example, by a Euclidean path integral or by Noether charges [see, e.g., [14]], and it is given by

$$
S_{\mathrm{BTZ}}^{\mathrm{st}}=4 \pi r_{+} .
$$

These computations do not depend only on the metric but also on the action, that is usually taken to be the standard action, hence the superscript st. Originally, this result comes from making geometrical considerations on the standard action of $2+1$ gravity, and then deriving the entropy from the grand canonical partition function in the classical approximation [15]

$$
Z=\exp \left(I_{\mathrm{st}}\right)
$$

Since the standard action is recovered from TMT, we can expect that the entropy of a BTZ black hole described by such an action can be recovered as well. Additionally, as we have shown, both the standard and exotic actions are derivable from TMT, thus we can also explore the entropy for the exotic BTZ black holes. Applying to the exotic action the same techniques that lead to Eq. (41), it is found that the entropy is proportional to the inner BTZ horizon, $r_{-}$. The fact that the entropy is proportional to the inner horizon raised doubts about the validity of black hole thermodynamics. However, it has been shown that these laws hold [3]. Indeed, the result is even more general: an entropy of the form

$$
S \sim \alpha r_{+}+\gamma r_{-}
$$

is in agreement with black hole thermodynamics. One advantage of the framework we are studying in this work is that Eq. (42) arises naturally. Hitchin's partition function is defined in terms of the volume functional,

$$
Z_{H}(\Phi)=\int_{[\Phi]} d \Phi \exp \left(V_{H}(\Phi)\right)
$$

Thus, when we write TMT as a theory of a $4 \mathrm{~d}$ vector bundle over a $3 \mathrm{~d}$ base space such that the $7 \mathrm{~d}$ manifold $X$ has a $G_{2}$ structure, we can separate $V_{7}$ in terms of the volume functionals $V^{ \pm}$,

$$
\lambda V_{7}=\beta_{+} V^{+}+\beta_{-} V^{-},
$$

for some coefficients $\lambda, \beta_{ \pm}$. Notice that, so far, all the properties that hold for a theory based on $V_{7}$ hold for a theory based on a multiple $\lambda$ of $V_{7}$. In addition, $V^{ \pm}$are proportional to the Chern-Simons actions, Eq. (32), with proportionality constants $1 / h^{ \pm}$. Putting all together, we write Hitchin's partition function as

$$
Z_{H}(\Phi)=\int_{[\Phi]} d \Phi \exp \left[\sum_{\sigma=+,-} \beta_{\sigma}\left(h^{\sigma}\right)^{-1 \sigma} I\right] .
$$

As before, the basis of the $7 \mathrm{~d}$ manifold can be decomposed into a $3 \mathrm{~d}$ base space and a $4 \mathrm{~d}$ bundle. The coefficients $\beta_{ \pm}$ can be chosen in such a way that the linear combination of ${ }^{ \pm} I$ in the argument of the exponential reproduces either the standard or the exotic action, or a combination of both. For the choice that leads to the standard action, by the discussion above we confirm that Hitchin's entropy is related to the BTZ entropy,

$$
Z_{H}(\Phi) \propto \int d e d \alpha \exp \left(I_{\mathrm{st}}\right)=Z_{\mathrm{BH}} .
$$


On the other hand, for a different choice of parameters we can have

$$
Z_{H}(\Phi) \propto \int d e d \alpha \exp \left(I_{\mathrm{ex}}\right)=Z_{\mathrm{BH}}
$$

i.e., the Hitchin partition function for the exotic action is also related to a black hole partition function, only that in this case $Z_{\mathrm{BH}}$ corresponds to the exotic BTZ black hole.

The extremal case, $r_{+}=r_{-}$, admits an interpretation from the point of view of TMT. Suppose we fix $\lambda$, e.g., $\lambda=1$. This imposes a constraint on the linear combinations in Eq. (44), such that any choice of $\beta_{ \pm}$leads to a fixed $V_{7}$ and the same $Z_{H}(\Phi)$. Accordingly all combinations lead to the same black hole entropy, and this is only possible if $r_{+}=r_{-}$, i.e., the extremal case corresponds to a constraint on the parameters $\beta_{ \pm}$.

\section{DISCUSSION}

Three-dimensional gravity can be embedded in a 7-manifold with $G_{2}$ holonomy. The volume form of this manifold is constructed in terms of a stable (generic, in the sense of [5]) form. Indeed, there are essentially two unique such forms and by using these two stable forms, we split the volume of the 7-manifold into contributions from the distinct orbits. Using the structure equations appropriated for our geometrical setup, we find that these two contributions can be rephrased as Chern-Simons actions, one for a self-dual curvature and one for an anti-self-dual curvature. This observation allows us to recover the two classically equivalent known actions of $3 \mathrm{~d}$ gravity, i.e., Witten's standard and exotic actions, thus completing the picture shown in $[5,12]$.

In a context that is more general than the theory that we study here, it has been conjectured that topological and black hole partition functions are related. Our results give a concrete realization of this conjecture: by writing the action of TMT in terms of the contributions from the two unique stable forms, we can tune the theory so that it reproduces the partition function of the standard action of 3d gravity, thus agreeing with the result for the BTZ black hole; or we can choose to reproduce the exotic action, obtaining the correct entropy for the exotic BTZ black hole. It is worth noticing that a combined standard/exotic entropy is in agreement with black hole thermodynamics [3], and our results provide a scenario where such combined models can be embedded. Moreover, any other action that can be written in terms of the standard and exotic action can be reinterpreted as a combination of the volume forms $V^{ \pm}$, and its Hitchin's partition function can be expressed as in Eq. (45). This is the case, for instance, for topologically massive ADS gravity [16], which also admits a BTZ black hole as an exact solution.

The topological partition function is also conjectured to be related to a wave function. The wave function for a static
BTZ black hole in the region outside the horizon has been computed within a canonical quantization scheme [9]. When evaluated at the horizon, their result takes the form (more details in the Appendix)

$$
|\psi|^{2} \sim e^{\tilde{\mu} r_{+}},
$$

where $\tilde{\mu}$ is a quantized number related to the energy levels of the system. This result indeed reassembles the Euclidean partition function for the BTZ black hole. It would be interesting to explore the quantization of a nonstatic BTZ black hole, so that the relation between the wave function and the black hole partition function can be explored for the extremal case, i.e., the case that would correspond to the conjectures in [8]. This is left for future work.

\section{ACKNOWLEDGMENTS}

This work is supported by CONACYT Grants No. 257919 and No. 258982. M. S. is supported by Convocatoria Institucional de Investigación Científica de la Universidad de Guanajuato (CIIC).

\section{APPENDIX: STATIONARY STATES OF THE BTZ BLACK HOLE}

In this appendix we briefly present the derivation done in [9], of the nonrotating BTZ black hole wave function

$$
\Psi=e^{(i / 4 G) \int_{0}^{\infty} d r \Gamma(r) W(\tau(r), R(r), F(r))},
$$

where $\tau=\tau(0), R=R(0)$, and $F=F(0)$. The WheelerDeWitt (WDW) equation becomes the Klein-Gordon (KG) equation

$$
\left[\frac{\partial^{2}}{\partial \tau^{2}}+F \frac{\partial^{2}}{\partial R^{2}}+A \frac{\partial^{2}}{\partial R}+B\right] e^{i \mu W(\tau, R, F)}=0 .
$$

As this description is based on a collapsing shell we impose that we have a free wave function; this is a natural assumption. For this to happen we should be able to write the WDW equation as

$$
\gamma^{a b} \nabla_{a} \nabla_{b} \Psi=0
$$

where $\gamma^{a b}$ is the DeWitt supermetric on the configuration space and $\nabla_{a}$ is the covariant derivative. The WDW equation is the free $\mathrm{KG}$ equation if $B=0$ and $A(R, F)=$ $|F| \partial_{R} \ln \sqrt{|F|}$ and the inner product is given by

$$
\left\langle\Psi_{1}, \Psi_{2}\right\rangle=\int \frac{d R}{\sqrt{|F|}} \Psi_{1}^{*} \Psi_{2} .
$$

When $F \neq 0$, the supermetric can be written in a flat form by the transformation $R_{*}= \pm \int|R|^{-1 / 2} d R$. In terms of $R_{*}$ the $\mathrm{KG}$ equation is 


$$
\left[\frac{\partial^{2}}{\partial \tau^{2}} \pm \frac{\partial^{2}}{\partial R_{*}^{2}}\right] e^{i \mu W(\tau, R, F)}=0
$$

the positive sign is for the exterior and the minus sign for the interior. The solutions are

$$
\begin{array}{cc}
\psi^{\text {in }}\left(\tau, R_{*}\right)=A_{ \pm} e^{-i \mu\left(\tau \pm R_{*}\right)} & F<0, \\
\psi^{\text {out }}\left(\tau, R_{*}\right)=B_{ \pm} e^{-i \mu\left(\tau \pm i R_{*}\right)} & F>0 .
\end{array}
$$

In the exterior

$$
R_{*}=\frac{1}{\sqrt{\Lambda}}\left[\ln \left(\frac{R \sqrt{\Lambda}+\sqrt{\Lambda R^{2}-8 G M}}{\sqrt{8 G M}}\right)+\frac{\pi}{2}\right]
$$

at the horizon $R_{*}=\ln r_{+}$. For a continuous wave function the matching conditions give the following spectrum:

$$
\mu_{j}=\sqrt{\Lambda} \hbar\left(j+\frac{1}{2}\right), \quad j=0,1,2, \ldots
$$

a similar spectrum was derived in [17].
[1] E. Witten, $(2+1)$-dimensional gravity as an exactly soluble system, Nucl. Phys. B311, 46 (1988).

[2] M. Banados, C. Teitelboim, and J. Zanelli, The Black Hole in Three Dimensional Space Time, Phys. Rev. Lett. 69, 1849 (1992).

[3] P. K. Townsend and B. Zhang, Thermodynamics of Exotic Bañados-Teitelboim-Zanelli Black Holes, Phys. Rev. Lett. 110, 241302 (2013).

[4] H. García-Compeán, O. Obregón, C. Ramírez, and M. Sabido, Remarks on $2+1$ self-dual Chern-Simons gravity, Phys. Rev. D 61, 085022 (2000).

[5] R. Dijkgraaf, S. Gukov, A. Neitzke, and C. Vafa, Topological M-theory as unification of form theories of gravity, Adv. Theor. Math. Phys. 9, 603 (2005).

[6] H.-V. Le, M. Panak, and J. Vanzura, Manifolds admitting stable forms, arXiv:0704.3894.

[7] J. Chagoya and M. Sabido, Topological M-theory, self-dual gravity and the Immirzi parameter, Classical Quantum Gravity 35, 165002 (2018).

[8] H. Ooguri, A. Strominger, and C. Vafa, Black hole attractors and the topological string, Phys. Rev. D 70, 106007 (2004).
[9] C. Vaz, S. Gutti, C. Kiefer, T. P. Singh, and L. C. R. Wijewardhana, Mass spectrum and statistical entropy of the BTZ black hole from canonical quantum gravity, Phys. Rev. D 77, 064021 (2008).

[10] R. L. Bryant, Metrics with exceptional holonomy, Ann. Math. 126, 525 (1987).

[11] A. Clarke and B. Santoro, Holonomy groups in Riemannian geometry, arXiv:1206.3170.

[12] R. L. Bryant and S. M. Salamon, On the construction of some complete metrics with exceptional holonomy, Duke Math. J. 58, 829 (1989).

[13] N. Hitchin, Stable forms and special metrics, arXiv:math/ 0107101.

[14] S. Carlip, The $(2+1)$-dimensional black hole, Classical Quantum Gravity 12, 2853 (1995).

[15] M. Banados, C. Teitelboim, and J. Zanelli, Black Hole Entropy and the Dimensional Continuation of the GaussBonnet Theorem, Phys. Rev. Lett. 72, 957 (1994).

[16] S. Carlip, S. Deser, A. Waldron, and D. K. Wise, Topologically massive AdS gravity, Phys. Lett. B 666, 272 (2008).

[17] A. Strominger, Black hole entropy from near horizon microstates, J. High Energy Phys. 02 (1998) 009. 